\title{
Neural and Behavioral Correlates of Alcohol-Induced Aggression Under Provocation
}

\author{
Gabriela Gan ${ }^{1,2}$, Philipp Sterzer ${ }^{3}$, Michael Marxen', Ulrich S Zimmermann' and Michael N Smolka ${ }^{*, 1}$ \\ 'Department of Psychiatry and Neuroimaging Center, Technische Universität Dresden, Dresden, Germany; ${ }^{2}$ Department of Psychiatry, Icahn \\ School of Medicine at Mount Sinai, New York, NY, USA; ${ }^{3}$ Department of Psychiatry and Psychotherapy, Campus Charité Mitte, Charité - \\ Universitätsmedizin Berlin, Berlin, Germany
}

\begin{abstract}
Although alcohol consumption is linked to increased aggression, its neural correlates have not directly been studied in humans so far. Based on a comprehensive neurobiological model of alcohol-induced aggression, we hypothesized that alcohol-induced aggression would go along with increased amygdala and ventral striatum reactivity and impaired functioning of the prefrontal cortex (PFC) under alcohol. We measured neural and behavioral correlates of alcohol-induced aggression in a provoking vs non-provoking condition with a variant of the Taylor aggression paradigm (TAP) allowing to differentiate between reactive (provoked) and proactive (unprovoked) aggression. In a placebo-controlled cross-over design with moderate alcohol intoxication $(\sim 0.6 \mathrm{~g} / \mathrm{kg}), 35$ young healthy adults performed the TAP during functional magnetic resonance imaging ( $\mathrm{M} M \mathrm{RI})$. Analyses revealed that provoking vs non-provoking conditions and alcohol vs placebo increased aggression and decreased brain responses in the anterior cingulate cortex/dorso-medial PFC (provoking < non-provoking) and the ventral striatum (alcohol<placebo) across our healthy sample. Interestingly, alcohol specifically increased proactive (unprovoked) but not reactive (provoked) aggression (alcohol $\times$ provocation interaction). However, investigation of inter-individual differences revealed (I) that pronounced alcohol-induced proactive aggression was linked to higher levels of aggression under placebo, and (2) that pronounced alcohol-induced reactive aggression was related to increased amygdala and ventral striatum reactivity under alcohol, providing evidence for their role in human alcohol-induced reactive aggression. Our findings suggest that in healthy young adults a liability for alcohol-induced aggression in a non-provoking context might depend on overall high levels of aggression, but on alcohol-induced increased striatal and amygdala reactivity when triggered by provocation.
\end{abstract}

Neuropsychopharmacology (20I5) 40, 2886-2896; doi:I0.I038/npp.20I5.I4I; published online 24 June 2015

\section{INTRODUCTION}

Aggression is an innate human behavior that can escalate to physical injury. However, people need to control possible outbursts of aggression in everyday life, because most societies will punish and not tolerate overt aggression. Behavioral control of aggression might especially be difficult under the influence of alcohol, a substance that impairs inhibitory control (eg, de Wit et al, 2000; Gan et al, 2014). Indeed, alcohol is involved in about one-half of violent crime, including domestic violence (Bureau of Justice Statistics, 1998; Foran and O'Leary, 2008; Bundeskriminalamt, 2011).

Experimental research has confirmed that alcohol increases aggression in men and women (eg, Taylor and Gammon, 1975; Dougherty et al, 1999; Giancola and Parrott, 2008). However, alcohol-induced aggression only occurs in a minority of people (Miczek et al, 1998; Heinz et al, 2011)

*Correspondence: Dr MN Smolka, Section of Systems Neuroscience, Department of Psychiatry and Psychotherapy, Technische Universität Dresden, Würzburger Street 35, Dresden 01 187, Germany,

Tel: +49 35I 463 4220I, Fax: +49 35I 46342202 ,

E-mail: michael.smolka@tu-dresden.de

Received 10 September 2014; revised I May 2015; accepted 5 May

2015; accepted article preview online I4 May 2015 with a predisposition for aggression (Parrott and Zeichner, 2002; Giancola et al, 2005) and is often triggered by provocation or threat (Taylor et al, 1976; Cherek et al, 1985).

A comprehensive neurobiological model (Heinz et al, 2011) suggests that alcohol-induced aggression might underlie diminished top-down control indicated by attenuated PFC activation and increased reactivity of the amygdala and ventral striatum under alcohol. Accordingly, alcohol-induced impairment of inhibitory control has been related to diminished activity of the lateral PFC (Kareken et al, 2013; Gan et al, 2014) and anterior cingulate cortex (ACC) under alcohol (Anderson et al, 2011). Aggressive behavior has also been linked to PFC hypo-activation, especially in the dorsal (d) ACC (Sterzer et al, 2005; Meyer-Lindenberg et al, 2006; Aharoni et al, 2013), and to increased activation of the amygdala, in particular for impulsive aggression triggered by provocation or threat, also referred to as reactive aggression (Meyer-Lindenberg et al, 2006; Coccaro et al, 2007). Reduced functional PFC-amygdala coupling under alcohol (Gorka et al, 2013) further suggests that alcohol-induced aggression might be mediated by impaired PFC functioning under alcohol, which might indirectly increase activation of the amygdala (Heinz et al, 2011). 
Moreover, alcohol might exert its effects on aggression by increasing dopamine levels in the ventral striatum (Dichiara and Imperato, 1986; Boileau et al, 2003; Heinz et al, 2011), a potential neural mechanism underlying experimentally induced aggression in animals (Miczek et al, 2002; Ferrari et al, 2003; Nelson and Trainor, 2007) and humans (Krämer et al, 2011; Beyer et al, 2014). Using fMRI, alcohol-induced increased dopamine levels might be reflected by increased activation of the ventral striatum in humans (cf, Gilman et al, 2008). However, the interaction between alcohol intoxication and aggression is complex and likely involves other neurotransmitters than dopamine such as serotonin and gamma amino-butyric acid (GABA) (Miczek et al, 2002; Seo et al, 2008; Heinz et al, 2011).

So far, the neural underpinnings of alcohol-induced aggression have mainly been studied in animal models of excessive aggression (eg, resident-intruder paradigms), which might not be representative for humans (Miczek et al, 2013). To the best of our knowledge, the present study investigates for the first time the neural mechanisms of alcohol-induced human aggression in relation to provocation at moderate alcohol intoxication (breath alcohol concentration $(\mathrm{BrAC})=0.6 \mathrm{~g} / \mathrm{kg})$. Healthy adults performed a variant of the TAP (Taylor, 1967; Krämer et al, 2007) with the aim to avoid potentially aversive punishments (ie, electric shocks). Participants played an equal amount of trials against a provoking and a non-provoking fictitious opponent who chose predominantly high aversive $v s$ low nonaversive punishments. Here, punishment intensity chosen by participants for their opponents can be considered as a measure of 'reactive' aggression in the provoking condition and 'proactive' or 'unprovoked' aggression in the nonprovoking condition.

On the neural level, we studied alcohol and provocation effects on brain responses during evaluation of opponents at the beginning of a trial to tackle the initial brain reactivity of being confronted with a provoking opponent compared with a non-provoking one, a process directly preceding the choice of the potentially aggressive punishment. We focused on regions of interest (ROIs) previously implicated in alcoholinduced aggression (cf, Heinz et al, 2011), including the ventral striatum, the amygdala, and the medial PFC/ACC, due to its role in evaluation and social cognition (Van Overwalle, 2009; Etkin et al, 2011). We expected that a

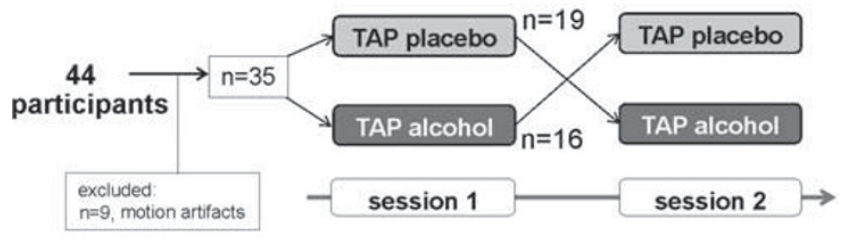

b

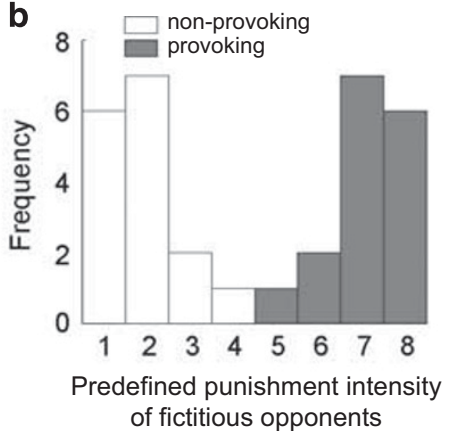

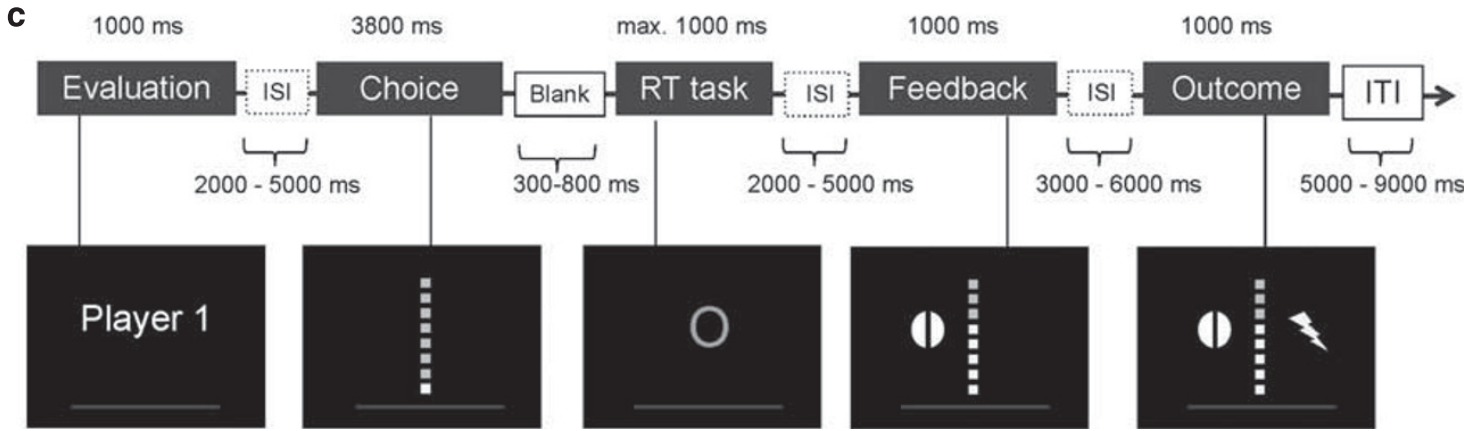

Figure I Study design and Taylor aggression paradigm (TAP). (a) Placebo-controlled, single-blind, cross-over study design. All participants performed the TAP once under alcohol and once under placebo, the order was counterbalanced across participants. (b) Distribution of predefined punishment intensities of the two fictitious opponents. The non-provoking opponent 'chose' mostly low intensities. The provoking opponent 'chose' mostly high intensities. (c) Timing and events for each experimental phase of the TAP (modified from Krämer et al, 2007). Evaluation: Presentation of the opponent for the upcoming trial by displaying 'Player I' or 'Player 2'. Opponents were color coded with a blue or red line displayed at the bottom of the screen (colors were counterbalanced across participants). Choice: Participants chose the punishment intensity from I to 8 for their opponent by pressing a button as soon as an upwards (starting from level I) or downwards (starting from level 8) moving indicator (a change of the color box from grey to yellow) on a vertical scale would reach the desired punishment level. Starting from top or bottom of the scale was equally distributed across trials/opponent. The first punishment intensity (I or 8 ) was displayed for $1000 \mathrm{~ms}$, all other levels for $400 \mathrm{~ms}$. After choosing a punishment intensity, the scale remained on the screen for the remaining time of the choice phase. If participants did not choose a punishment intensity, they automatically lost that trial. Reaction time (RT) task: Participants had to press a button as fast as possible when a green circle appeared on the screen. If people reacted within $1000 \mathrm{~ms}$, the circle changed its color to yellow (indicating a response in time but did not inform about winning or losing); otherwise the green circle disappeared after 1000 ms and participants would automatically lose the trial. Feedback and outcome: Circles with a vertical ('lost', as shown in panel c) and horizontal bar ('won') indicated whether participants won or lost the RT task. The punishment intensity that would either be administered to themselves in lost trials or to their opponent in won trials in the outcome phase was displayed by means of the vertical scale. Outcome: a yellow flash indicated administration of the electric shock, which was applied to the participant with the indicated punishment intensity in lost trials for $50 \mathrm{~ms}$ at the beginning of the outcome phase. A white fixation cross on a black background was shown in inter-stimulus intervals (ISIs) and inter-trial intervals (ITIs). A full colour version of this figure is available at the Neuropsychopharmacology journal online. 
provocation (provoking $v s$ non-provoking condition) would go along with increased aggression and diminished ACC/ medial PFC activity as previously observed in aggressive populations. For alcohol vs placebo, we expected increased aggression and diminished PFC activation as well as increased ventral striatum and amygdala reactivity. Bearing in mind the inter-individual effect of alcohol on aggression, we computed brain-behavior correlations to test whether specifically people with pronounced alcohol-induced reactive aggression elicited by provocation, a potentially threatening and stressful situation, might exhibit increased amygdala and ventral striatum reactivity under alcohol (Heinz et al, 2011).

\section{MATERIALS AND METHODS}

\section{Participants}

Forty-four young social drinkers $(\geqslant 2$ drinks/week, $\geqslant 1$ lifetime occasion of getting drunk), included in the Dresden Longitudinal study on Alcohol Use in Young Adults (DLAYA), performed the TAP twice on separate days during fMRI (Figure 1a). All participants were physically (eg, no central nervous, cardio-vascular, or liver/kidney disease) and mentally healthy (eg, no previous or current alcohol/illicit drug abuse/dependence), abstained from illicit drugs/alcohol for at least 1 week/24 h before each experimental day, had a positive $(\mathrm{FH}+$, at least one first-degree biological relative affected by alcoholism) or negative (no biological relative affected by alcoholism) family history of alcoholism (FHA; considered as covariate if affecting outcome variables) (for recruitment, detailed inclusion/exclusion criteria: Marxen et al, 2014; Gan et al, 2014). Exclusion criteria were MR-contraindications and pregnancy or breast-feeding in women.

The final sample for fMRI and behavioral data analysis consisted of 35 participants (mean age $=19.1$ years, $\mathrm{SD}=0.4$; $12 \mathrm{FH}+, 11$ female; $n=9$ excluded due to extensive head movements during $\mathrm{fMRI}:>3 \mathrm{~mm} /{ }^{\circ}$ translation/rotation) characterized by moderate-to-high alcohol consumption, low trait aggression, and low impulsivity (see Supplementary Table S1).

All participants provided written informed consent after complete description of the study. The D-LAYA study was approved by the Ethics Committee of the Technische Universität Dresden.

\section{Experimental Procedure}

On arrival, all participants were sober $(\mathrm{BrAC}=0.0 \mathrm{~g} / \mathrm{kg})$ and had negative test results for a urine screening of illicit drugs and pregnancy in women.

All study procedures, including alcohol administration, are detailed elsewhere (Marxen et al, 2014; Gan et al, 2014). For the placebo-controlled cross-over design, see Figure 1a. Alcohol (6\% alcohol solution, v/v; mixture of normal saline with 95\% ethanol (Braun, Melsungen, Germany)) and placebo (normal saline) were administered intravenously using computer-assisted alcohol infusion systems (O'Connor et al, 1998). On each experimental day, the TAP was performed approximately $75 \mathrm{~min}$ after starting the infusion during fMRI (Figure 1 in Marxen et al, 2014). Aggression measured with the TAP has been shown to increase linearly with alcohol dose in a range of $0.0-1.0 \mathrm{~g} / \mathrm{kg}$ (Duke et al, 2011). For the alcohol condition, we chose a moderate target $\mathrm{BrAC}$ level of $0.6 \mathrm{~g} / \mathrm{kg}$ (mean $\mathrm{BrAC}=0.63 \mathrm{~g} / \mathrm{kg} \pm 0.03 \mathrm{SD}$; no significant difference between BrAC pre/post-TAP: $0.64 \pm 0.04 / 0.63 \mathrm{~g} / \mathrm{kg} \pm 0.04 ; \quad$ paired $t$-test, $t(33)=1.37$, $P=0.18$ ) shown to fall on the ascending limb of human alcohol-induced aggression (Duke et al, 2011).

Additionally, we measured absolute perfusion directly after the TAP under alcohol and placebo with arterial spin labeling MRI to assess if alcohol-induced increased cerebral perfusion in the same sample (cf, Marxen et al, 2014) interacted with alcohol effects on task-related blood oxygenation level-dependent (BOLD) responses (for MRI data acquisition, see Supplementary Results).

\section{Taylor Aggression Paradigm}

In a modified fMRI version of the TAP (Krämer et al, 2007), participants played against one of the two fictitious players at a time in a competitive reaction time (RT) game, 32 trials against each (total $=64$ trials). The aim was to respond faster than the opponent to avoid a potentially aversive punishment (ie, an electric shock) administered to the losing player of the trial via an MR-compatible electrode to the left forearm (for equipment, see Supplementary Methods and Results). In each trial, participants chose the punishment intensity, ranging from 1 (non-aversive) to 8 (maximally aversive), for their opponent before performing the RT task. The chance of winning was $50 \%$ with the following exception: If participants responded $50 \%$ faster than their mean RT of past trials per opponent, they would win the trial; if they responded 50\% slower, they would lose the trial. Aggression was measured as the mean punishment intensity separately for the provoking and non-provoking condition, considered as reactive $v s$ proactive aggression. The RT from the RT task served as behavioral measure of motivation to win.

Each trial consisted of five consecutive events (for details, see Figure 1c): (1) presentation of the opponent for the upcoming trial (evaluation), (2) choice of the punishment intensity for the opponent, (3) RT task, (4) feedback about winning or losing the RT task, and (5) administration of the electric shock to the losing player (outcome).

Participants did not know that their opponents were fictitious and that the non-provoking opponent would predominantly choose low non-aversive punishments, and the provoking opponent would choose high aversive punishments (see Figure 1b, Supplementary Methods and Results for cover story). The sequence of trials against the two opponents was randomized. Participants won or lost maximally three times in a row per opponent. The TAP consisted of two 16-min runs with a 1-2 min break. We varied inter-stimulus and inter-trial intervals using uniform distributions (see Figure 1c). Minimum and maximum punishment intensities were determined individually before the TAP in the MR-scanner (see Supplementary Methods and Results).

Unlike the TAP version by Krämer et al (2007), we separated the evaluation from the choice phase and the feedback from the outcome phase (Figure 1c) and assessed brain responses during evaluation of opponents, a process not superimposed by motor activity that preceded the choice of a potentially aggressive response. 


\section{Data Analysis}

Behavioral data were analyzed with SPSS21 (IBM, NY, USA) and fMRI data with statistical parametric mapping (SPM8, Wellcome Trust Centre for Neuroimaging, London, UK).

\section{Behavioral Analysis}

We assessed drug and provocation effects on aggression and RTs using $2 \times 2$ repeated-measures ANOVAs with drug (2 levels: alcohol, placebo) and provocation (2 levels: provoking, non-provoking) as within-subject factors and drug order as between-subjects factor (2 levels: placebo first, alcohol first). Potentially confounding variables such as gender and FHA were not considered as covariates in the statistical analyses as they did neither affect drug nor provocation effects on aggression and RTs (see Supplementary Results). To explore inter-individual differences in alcohol-induced reactive aggression (provoking condition) and proactive aggression (non-provoking condition), we computed correlations with physical trait aggression measured with the Buss-Perry Aggression Questionnaire (BPAQ) (Buss and Perry, 1992; German version: Herzberg, 2003) and experimentally induced placebo aggression (measured by the TAP), variables known to affect alcohol-induced aggression (Dougherty et al, 1999; Giancola and Parrott, 2008).

\section{fMRI Analysis}

On the subject level, we modeled evaluation, choice, feedback, and outcome phases as separate events for each run of the placebo and alcohol session using a general linear model. We separately modeled brain responses of the provoking and non-provoking condition for all experimental phases and additionally of won and lost trials for the feedback and outcome phase. The onsets of events were convolved with SPM8's canonical hemodynamic response function. For each run, we included seven nuisance variables: motor responses (1: RT task, choice phase, and unintended responses), and motion parameters (6: 3 translations, 3 rotations). We corrected for serial auto-correlations using an autoregressive AR(1)-model (for MRI data preprocessing, see Supplementary Results).

On the second level, we assessed drug (alcohol-placebo) and provocation (provoking-non-provoking) effects on brain responses during evaluation of opponents by subjecting first-level contrasts of the evaluation phase for each provocation condition $v s$ baseline for placebo and alcohol to a $2 \times 2$ full-factorial model, with drug and provocation as within-subject factors. Specifically, we used small volume correction (SVC) to investigate provocation and drug effects in ROIs implicated in alcohol-induced aggression (see Figure 4a-right): bilateral ventral striatum, bilateral amygdala, and the medial part of the PFC including the ACC and dorsal/ventral medial $(\mathrm{dm} / \mathrm{vm})$ PFC due to their role in evaluation and aggression. Additionally, to explore neural correlates of evaluation measured with our modified TAP version in more depth, we computed whole-brain analyses using a liberal significance threshold of $P<0.001$ (uncorrected, $\geqslant 15$ connected voxels) to avoid type-II errors (Lieberman and Cunningham, 2009).

Second, to investigate neural correlates of individual levels of alcohol-induced reactive/provoked and proactive/unprovoked aggression (Miczek et al, 1998; Heinz et al, 2011), we computed brain-behavior correlations between interindividual levels of alcohol-induced changes of aggression and brain responses in ROIs. Alcohol effects were computed ('alcohol-placebo') separately for the provoking and nonprovoking condition based on extracted mean signal change (\%) in ROIs from first-level contrasts. We compared brainbehavior correlations between provocation conditions using Steiger's Z (Steiger, 1980; R-toolbox: Diedenhofen, 2013). For the amygdala and ventral striatum, we applied one-tailed significance testing because we expected increased reactivity
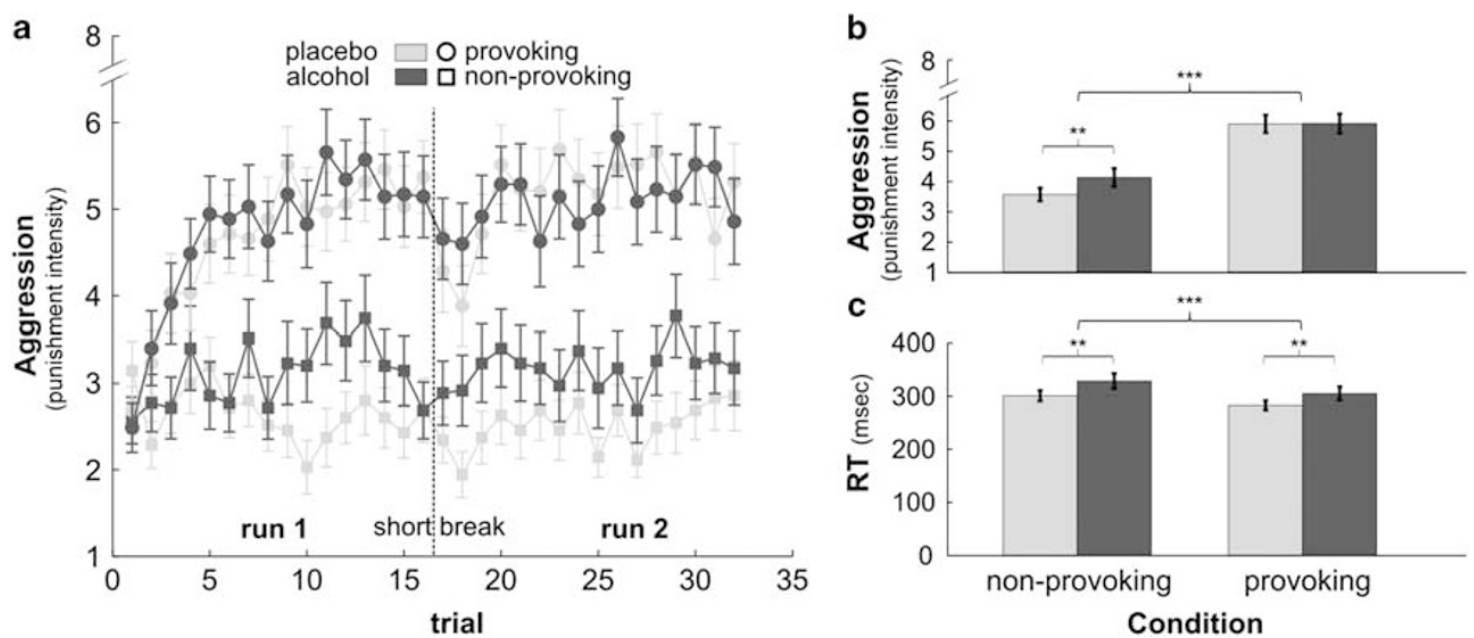

Figure 2 Alcohol and provocation effects on aggression and reaction times (RT; descriptive means \pm SEM). (a) Aggression averaged across participants is plotted for each experimental trial and each provocation and drug condition. After approximately five trials against each opponent, participants were more aggressive in the provoking than in the non-provoking condition. (b) Aggression averaged across trials was increased in the provoking compared with the nonprovoking condition $\left(F(1,33)=66.19, P<0.001, \eta^{2}=0.670\right)$, and under alcohol compared with placebo $\left(F(I, 33)=4.68, P=0.037, \eta^{2}=0.125\right)$. A drug $\times$ provocation interaction indicated that the main effect of alcohol was driven by the non-provoking condition $\left(F(1,33)=8.23, P=0.007, \eta^{2}=0.20\right)$. (c) Reaction times during the competitive RT task. Participants reacted faster in the provoking than in the non-provoking condition $(F(I, 33)=I 7.99, P<0.00 \mathrm{I}$, $\left.\eta^{2}=0.35\right)$, and alcohol significantly increased $R T s\left(F(I, 33)=\mid 2.81, P=0.00 I, \eta^{2}=0.28\right)$. ${ }^{*} * * P<0.00 I$, $* * P<0.005$. A full colour version of this figure is available at the Neuropsychopharmacology joumal online. 
under alcohol specifically for alcohol-induced reactive aggression (Heinz et al, 2011). We corrected $\alpha$ for number of ROIs.

Controlling the whole-brain analysis and brain-behavior correlations for drug order, gender, and FHA (Supplementary Table S2A-C,Supplementary Table S3Aright column), or for global perfusion under alcohol and placebo (Supplementary Table S2D and E,Supplementary Table S3A-middle column), did not affect BOLD responses substantially. Thus we did not include covariates into statistical models.

\section{RESULTS}

\section{Behavioral Data}

Participants reliably differentiated between the two opponents after approximately five trials/opponent with increased

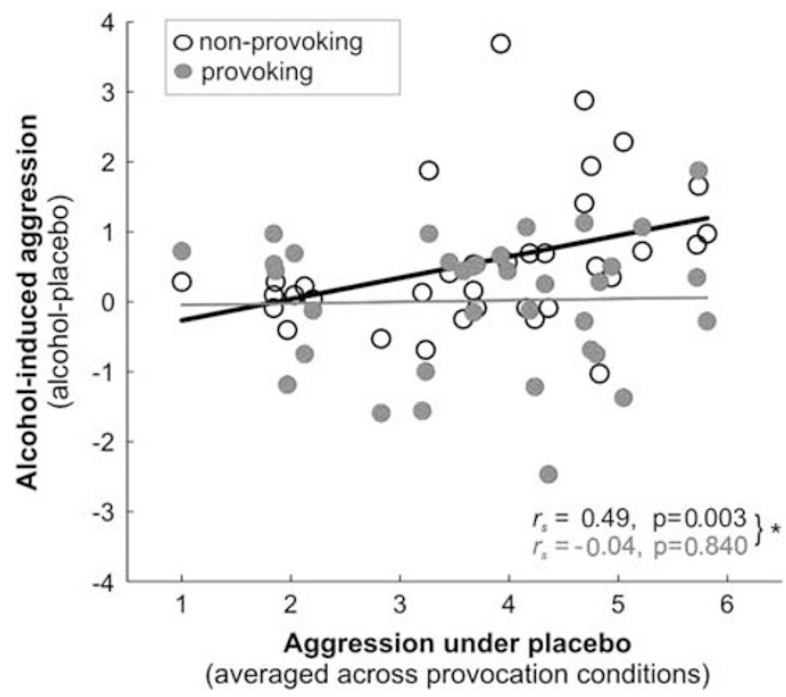

Figure 3 Correlation plots for mean aggression under placebo (averaged across provocation conditions) and alcohol-induced aggression in the provoking (red/grey circles) and non-provoking (white circles) condition. Higher levels of aggression under placebo went along with higher levels of alcohol-induced aggression in the non-provoking, but not in the provoking condition. $* P=0.007$ (two-tailed), significant difference between correlation coefficients of provocation conditions; $r_{s}=$ Spearman correlation coefficient. A full colour version of this figure is available at the Neuropsychopharmacology journal online. aggression under provocation, indicating a learning process (Figure $2 \mathrm{a}$ ). Separate $2 \times 2$ repeated-measures ANOVAs with drug and provocation as within-subject factors and drug order as between-subjects factor for aggression and RTs confirmed that provoking compared with non-provoking conditions (main effect of provocation, Figure $2 b$ and $c$ ) increased aggression $\left(\mathrm{F}(1,33)=66.19, P<0.001, \eta^{2}=0.670\right)$ and decreased RTs $\left(\mathrm{F}(1,33)=17.99, P<0.001, \eta^{2}=0.35\right)$. Alcohol vs placebo (main effect of drug, Figure $2 b$ and $c$ ) increased aggression $\left(\mathrm{F}(1,33)=4.68, P=0.037, \eta^{2}=0.125\right)$ and $\mathrm{RTs}\left(\mathrm{F}(1,33)=12.81, P=0.001, \eta^{2}=0.28\right)$. However, alcohol increased aggression in the non-provoking condition only, indicated by a drug $\times$ provocation interaction ( $F$ $(1,33)=8.23, P=0.007, \eta^{2}=0.20$, Figure $\left.2 b\right)$. Drug order did not affect aggression $(P>0.6)$, but RTs were overall faster on the second day irrespective of drug (drug $\times$ drug order interaction, $\left.\mathrm{F}(1,33)=51.57, P<0.001, \eta^{2}=0.61\right)$.

Correlation analyses revealed that higher levels of placebo TAP aggression (averaged across provocation conditions) were specifically linked to pronounced alcohol-induced proactive aggression (non-provoking condition, Spearman's $r=0.49, P=0.003$ ) but not reactive aggression (provoking condition, Spearman's $r=-0.04, P=0.83$ ). Correlation coefficients differed significantly between provocation conditions (Steiger's $Z=2.69, P=0.007$, Figure 3). However, physical trait aggression assessed with the BPAQ did neither affect alcohol-induced reactive (Spearman's $r=0.12, P=0.50$ ) nor proactive aggression (Spearman's $r=0.23, P=0.19$ ).

\section{Neuroimaging Data}

ROI and whole-brain analyses $(2 \times 2$ full-factorial model $)$ revealed decreased brain responses (Figure $4 \mathrm{a}$ and $\mathrm{b}$ ) during evaluation of the provoking $v s$ the non-provoking opponent in bilateral dACC/dmPFC (significant main effect of provocation following SVC with the ACC/medial PFC mask and across the whole brain, Table 1A) and for alcohol relative to placebo in the bilateral ventral striatum (significant main effect of alcohol following SVC with the ventral striatum mask; Table 1B). Exploratory whole-brain analyses (Table $1 \mathrm{~A}$ and $\mathrm{B}$, Figure $4 \mathrm{a}$ ) revealed that provocation and alcohol additionally decreased brain responses during evaluation (1) in the left temporo-parietal cortex, left thalamus, and right posterior insula for 'provoking $<$ non-provoking' conditions, and (2) in occipito-temporal and inferior parietal

Figure 4 Alcohol and provocation effects on brain responses during evaluation of opponents. (a) Left: Main effects of provocation (provoking < nonprovoking condition) and alcohol (alcohol<placebo) on brain responses during evaluation of opponents $(2 \times 2$ (Drug $\times$ Provocation) full-factorial wholebrain analysis). Decreased brain responses during evaluation of the provoking compared with the non-provoking opponent in the dorsal (d)ACC/dorso-medial (dm)PFC, temporo-parietal junction, left thalamus, and posterior insula (cyan color) and for alcohol compared with placebo in occipito-temporal and inferior parietal cortex, inferior/middle frontal areas, hippocampus, and bilateral ventral striatum (blue color). The whole-brain significance threshold was set to $P<0.00$ I uncorrected with 15 connected voxels. Brain areas highlighted in red represent clusters in ROls showing significant provocation-specific effects in $\mathrm{dACC/dmPFC}$ and alcohol-sensitive effects in bilateral ventral striatum following ROI analyses using SVC (FWE-correction at peak level). (a) Right: ROls implicated in alcohol-induced aggression. ACC/medial PFC mask and bilateral amygdala mask based on the AAL atlas (Maldjian et al, 2003), bilateral ventral striatum defined by meta-analysis on reward processing (Liu et al, 20I I) (I0-mm spheres around MNI peak coordinates $(x= \pm \mid 2, y=10, z=-6)$ ). (b) Mean signal change in alcohol-sensitive bilateral ventral striatum and provocation-specific dACC/dmPFC (extracted from clusters surviving the whole-brain threshold of $P<0.001$ with $\geqslant 15$ connected voxels), and additionally in the amygdala (extracted from anatomical mask). Error bars indicate SEM. (c) Brain-behavior correlations between alcohol-induced aggression and alcohol-induced changes of brain responses (alcohol-placebo) differ significantly between provocation conditions in the alcohol-sensitive ventral striatum and in the amygdala (one-tailed $P$-values). Pronounced alcohol-induced aggression is linked to increased reactivity of the ventral striatum and amygdala under alcohol in the provoking condition only. No significant brain-behavior correlations were observed for the non-provoking condition and in the dACC/dmPFC. $r=$ Pearson correlation coefficient; $* * P=0.004$ (one-tailed); $* P=0.02$ (one-tailed), ' $P=0.3 \mathrm{I}$ (two-tailed). 
a
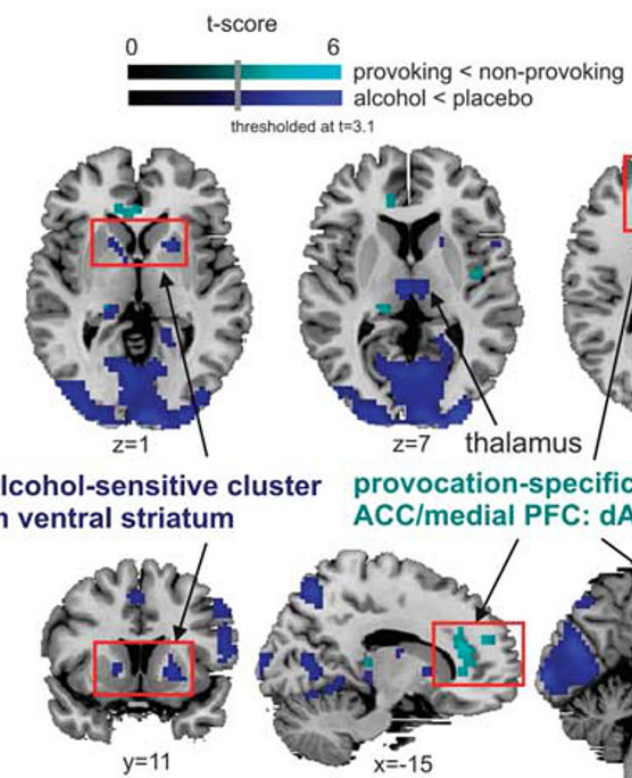

$z=7$ thalamus

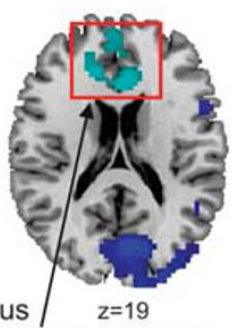

provocation-specific cluster in ACC/medial PFC: dACC/dmPFC
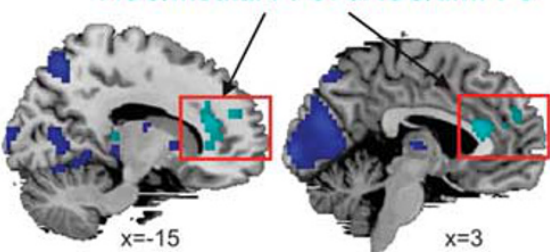

provocation-specific ROI: dACC/dmPFC
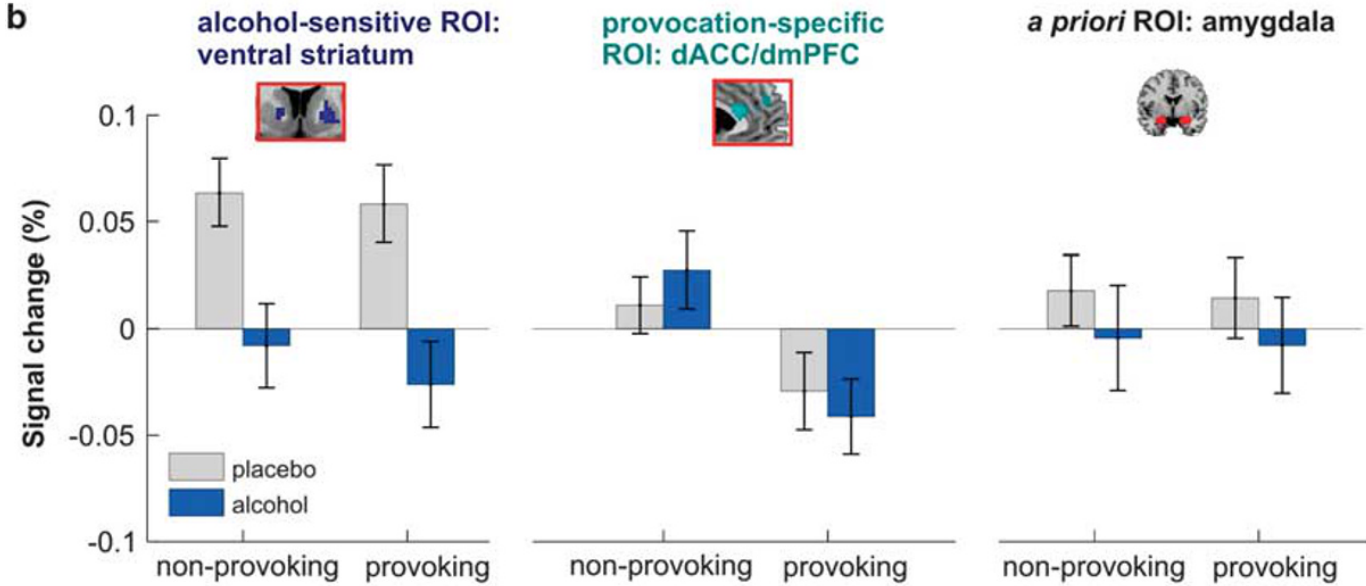

non-provoking provoking

Condition

c

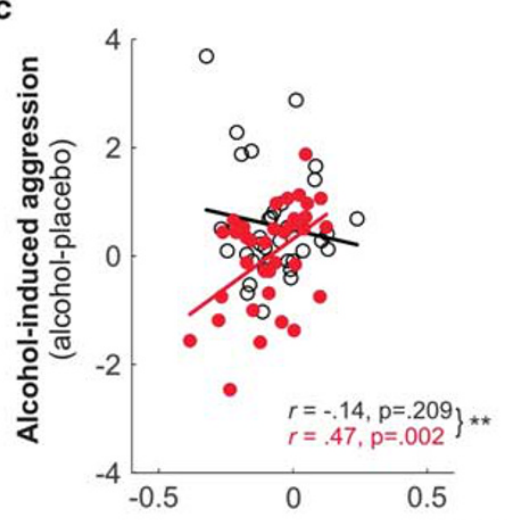

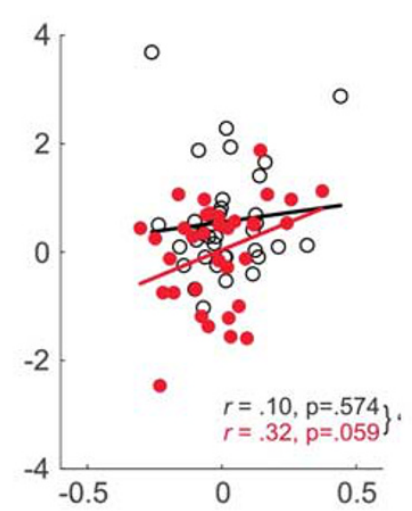

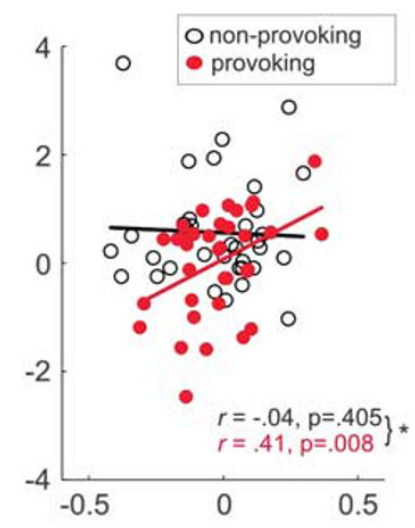

Alcohol-induced changes of brain responses

(alcohol-placebo, signal change in \%)

cortex, right inferior/middle frontal areas, and bilateral hippocampus for 'alcohol< placebo'.

Across all participants, neither ROI nor whole-brain analyses revealed alcohol effects in the ACC/medial PFC $\left(t_{s}<3.0, p_{s}>0.5\right)$ nor alcohol and provocation effects in the amygdala $\left(t_{s}<2.7, p_{s}>0.2\right)$. No brain area exhibited increased activation for 'provoking $>$ non-provoking' conditions or 'alcohol>placebo'. We did not further explore a 
'drug $\times$ provocation' interaction in the superior PFC (Table 1C) as it did not belong to our ROIs.

To investigate the relationship between alcohol effects on aggression and brain responses in ROIs, we defined a provocation-specific prefrontal ROI (dACC/dmPFC), and alcohol-sensitive ventral striatum ROI based on the wholebrain group analysis (significance threshold: $P<0.001$ (uncorrected), $\geqslant 15$ connected voxels) to target task-related brain areas (Figure $4 \mathrm{~b}$ for contrast estimates). We collapsed brain responses across the right and left ventral striatum as alcohol effects did not differ between hemispheres $(t(34)=0.003, P=0.99)$. Due to a strong a priori hypothesis, we also computed brain-behavior correlations for the amygdala, regardless of missing main effects of provocation or alcohol across participants.

Brain-behavior correlations in ROIs revealed that alcoholinduced changes of aggression and brain responses differed significantly between provoking and non-provoking conditions in the alcohol-sensitive ventral striatum (Steiger's $Z=2.63, P=0.004$, one-tailed) and marginally significantly in the amygdala (Steiger's $Z=2.03, P=0.02$, one-tailed; corrected $\alpha$-level: $P<0.017)$. Thus alcohol-induced reactive aggression (provoking condition) correlated positively with alcohol-induced increased reactivity of the ventral striatum (Pearson's $r=0.47, P=0.002$, one-tailed; equivalent results for a priori ventral striatum mask: Supplementary Table S3B) and the amygdala (Pearson's $r=0.41, P=0.008$, one-tailed; Figure 4c). No correlation with alcohol-induced aggression was observed in the dACC/dmPFC and the amygdala and ventral striatum for the non-provoking condition (Figure 4c).

Additionally, we observed that increased ventral striatum and amygdala reactivity for the 'provoking $>$ non-provoking' condition (averaged across drug) was associated with increased aggression in the provoking relative to the nonprovoking condition (amygdala: Pearson's $r=0.36, P=0.035$; ventral striatum: Pearson's $r=0.39, P=0.019$ ).

\section{DISCUSSION}

Using a multi-modal assessment of alcohol-induced aggression, including behavioral, neuroimaging, and self-report measures, in a placebo-controlled cross-over design, we observed that neural and behavioral correlates of alcoholinduced aggression in young healthy adults vary as a function of provocation. Pronounced alcohol-induced aggression measured with the TAP was linked to alcoholinduced increased striatal and amygdala reactivity in a provoking context and to higher levels of placebo TAP aggression but not alcohol-induced changes of brain activation in a non-provoking situation.

Results were characterized by robust behavioral and neural provocation effects indicated by increased aggression, faster RTs, and decreased dACC/dmPFC activity during evaluation of the provoking compared with the non-provoking opponent. These provocation effects, especially in medial prefrontal brain areas implicated in evaluation (Etkin et al, 2011) and social cognition (eg, Van Overwalle, 2009), demonstrate face validity of the modified TAP version by suggesting that participants were engaged in the game and evaluated the two opponents differently. Faster
RTs in the provoking condition indicate that participants made a larger effort in the belief that they might avoid the aversive punishment (although the chance of winning was $50 \%$ ). Attenuation of $\mathrm{dACC} / \mathrm{dmPFC}$ activity in the provoking condition that elicited increased aggression is consistent with dACC dysfunction in aggressive populations (Sterzer et al, 2005; Meyer-Lindenberg et al, 2006; Aharoni et al, 2013).

Alcohol is suggested to increase aggression in response to provocation (Giancola and Parrott, 2008). However, across our healthy young sample alcohol did not increase 'reactive' aggression provoked by high-intensity provocations but specifically 'proactive' aggression in the non-provoking situation (non-aversive punishments). We doubt that the missing alcohol effect under provocation can be attributed to a ceiling effect as reactive aggression was considerably below the maximum intensity of ' 8 ' (alcohol/placebo mean \pm SD: $4.9 / 4.9 \pm 1.7 / 1.9$ ). Possibly, becoming aggressive in response to high-intensity provocations occurs irrespective of alcohol because the threshold for aggressive behavior might even be low under placebo when provocation is high. Our data suggest that in healthy adults alcohol may facilitate aggression more potently in low provoking situations, such as repeated poking in a bar. However, alcohol-induced aggression only occurs in a minority of people and might especially manifest in individuals with high aggression traits (Miczek et al, 1998; Heinz et al, 2011).

Indeed, examination of inter-individual differences of alcohol-induced aggression revealed that pronounced alcohol-induced proactive, but not reactive aggression, was related to high levels of placebo TAP aggression (Dougherty et al, 1999). While alcohol-induced proactive aggression might depend on a disposition for aggressive behavior, alcohol-induced reactive aggression might occur regardless of overall aggression levels and alcohol intoxication when provocation is high enough. However, self-reported trait aggression was neither related to alcohol-induced reactive nor proactive aggression, probably due to overall low trait aggression in our sample. Here, expectancies about the effects of alcohol on aggression that are likely shaped by social learning (Bandura, 2001; cf, Heinz et al, 2011) might help to understand why some people get specifically aggressive under alcohol in low provoking situations, such as being poked in a bar or a domestic context (Foran and O'Leary, 2008).

Importantly, individuals with increased amygdala and ventral striatum reactivity under alcohol exhibited pronounced alcohol-induced reactive aggression when being provoked. This inter-individual provocation-specific effect on neural correlates of alcohol-induced aggression strengthens the idea that the amygdala and ventral striatum are especially involved in alcohol-heightened aggression in stressful and threatening situations, such as being provoked (cf., Heinz et al, 2011). Increased ventral striatum reactivity in individuals with high levels of alcohol-induced reactive aggression suggests an involvement of dopamine, which has been discussed to facilitate aggression by increasing arousal and stress (Miczek et al, 2002; Ferrari et al, 2003; Nelson and Trainor, 2007). However, the interplay between neurotransmitters such as serotonin, dopamine, and GABA in alcoholinduced human aggression is not well understood (Miczek et al, 2002; Seo et al, 2008; Heinz et al, 2011). 
Table I Alcohol and Provocation Effects on Brain Responses During Evaluation of Opponents $(2 \times 2$ Full-Factorial Model)

MNI coordinates

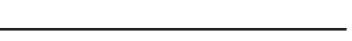

P-value

\begin{tabular}{llll}
\hline \multicolumn{2}{c}{ Cluster level } & & \multicolumn{2}{c}{ Peak level } \\
FWE-corr & Unc & FWE-corr & Unc
\end{tabular}

Brain area

BA

\begin{abstract}
$\mathbf{x}$
\end{abstract}
y k
$<0.00$ I

$\mathbf{R} \mathbf{A C C}$

L ACC

L SFG

L STG/Angular G

R Superior medFG

$\mathrm{L}$ Thalamus

$R$ post. Insula

$\mathrm{R}$ Insula

$\begin{array}{cc}24 & 3 \\ 32 & -15 \\ 9 & -15 \\ 39 & -48 \\ 8 & 12 \\ - & -18 \\ 13 & 48 \\ 13 & 42\end{array}$

26
35
47
-58
44
-31
-7
-7

19
16
22
25
40
7
13
7

268

.

$0.37 \mid$

$$
0.517
$$

0.637

0.481

28

$<0.001$

0.081

0.081

0.128

0.178

B: Alcohol<placebo

\begin{tabular}{|c|c|c|c|}
\hline R MOG & 19 & 27 & -97 \\
\hline R MOG & 18 & 18 & -103 \\
\hline L Fusiform G & 19 & -30 & -79 \\
\hline $\mathrm{L} I \mathrm{IPL}$ & 40 & -36 & -52 \\
\hline L Precuneus & 7 & -21 & -64 \\
\hline L Precuneus & 7 & -6 & -73 \\
\hline L Hippocampus & - & -21 & -28 \\
\hline L Parahipp. G & 36 & -24 & -31 \\
\hline L Culmen & - & -12 & -55 \\
\hline R MFG & 6 & 39 & 2 \\
\hline R MFG & 8 & 51 & 11 \\
\hline R Putamen ${ }^{b}$ & - & 18 & 11 \\
\hline R IFG & 44 & 57 & 11 \\
\hline L Thalamus & - & -3 & -19 \\
\hline R Thalamus & - & 6 & -16 \\
\hline L Thalamus & VLN & -12 & -10 \\
\hline R Culmen & - & 39 & -43 \\
\hline R Declive & - & 30 & -55 \\
\hline R Fusiform G & 19 & 27 & -76 \\
\hline R Fusiform G & 19 & 36 & -76 \\
\hline R Fusiform G & 19 & 30 & -67 \\
\hline R Hippocampus & 27 & 27 & -31 \\
\hline L Globus pallidus & I.Ncl. & -9 & -1 \\
\hline L Caudate ${ }^{c}$ & Head & -12 & 8 \\
\hline L SMA & 6 & -3 & | | \\
\hline
\end{tabular}

$10 \quad 2129$

$<0.00$ I

$<0.001$

0.005

0.008

0.008

$-17$

52

1139

$<0.001$

$<0.001$

0.036

0.037

0.040

$0.059 \quad 0.011$

85

-5
-14

$-11$

43

43

।

13

7

7

13

$-32$

32
-17

$-17$

$-17$

$-14$

$-5$

$-2$

4
49

86

0.057

0.010

0.415

0.156

0.094

0.030

0.081

0.015

76

66

0.116

0.022

0.145

0.027

60

0.680

0.680

0.702

0.200

0.616

0.212

0.168

0.575

73
67

$-33$

$-7$

L SFG
L Precentral G

$\begin{array}{ll}6 & -24 \\ 6 & -33\end{array}$

$-10$

67

0.062

0.116

0.573

0.490
0.727

0.908

0.919

0.929

$\begin{array}{ll}<0.001 & 4.66 \\ <0.001 & 4.47 \\ <0.001 & 3.87 \\ <0.001 & 3.95 \\ <0.001 & 3.73 \\ <0.001 & 3.50 \\ <0.001 & 3.48 \\ <0.001 & 3.47\end{array}$

$<0.001 \quad 5.35$

$<0.001 \quad 5.22$

$<0.001 \quad 5.21$

$<0.001 \quad 4.82$

$<0.001 \quad 4.81$

$<0.001 \quad 4.78$

$<0.001 \quad 4.53$

$\begin{array}{lll}0.726 & <0.001 & 3.73 \\ 0.875 & <0.001 & 3.55\end{array}$

$0.181<0.001 \quad 4.32$

$0.433<0.001 \quad 3.99$

$0.266<0.001 \quad 4.20$

$0.281<0.001 \quad 4.18$

$0.321<0.001 \quad 4.13$

$0.596<0.001 \quad 3.85$

$0.818<0.001 \quad 3.63$

$0.391<0.001 \quad 4.05$

$0.951<0.001 \quad 3.41$

$0.515<0.001 \quad 3.93$

$0.822<0.001 \quad 3.62$

$0.869<0.001 \quad 3.56$

$0.713<0.001 \quad 3.74$

$0.760<0.001 \quad 3.69$

$0.880<0.001 \quad 3.55$

$0.805 \quad<0.001 \quad 3.64$

Abbreviations: ACC, anterior cingulate cortex; BA, Brodmann area; G, gyrus; IFG, inferior frontal gyrus; IPL, inferior parietal lobe; I.Ncl., lentiform nucleus; medFG, medial

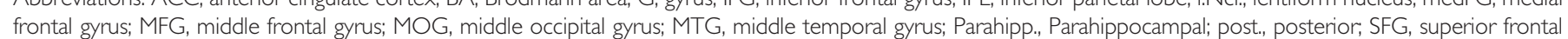
gyrus; SMA, supplementary motor area; STG, superior temporal gyrus; VLN, ventro-lateral nucleus.

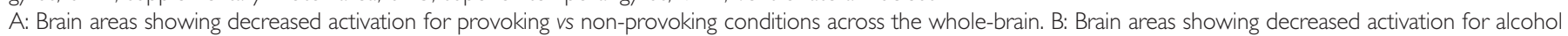

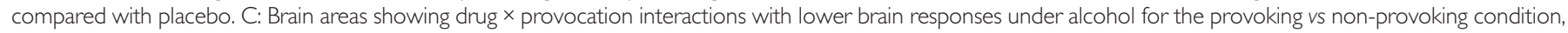
and no difference between provocation conditions under placebo. No brain area showed increased activation for the provoking compared with non-provoking

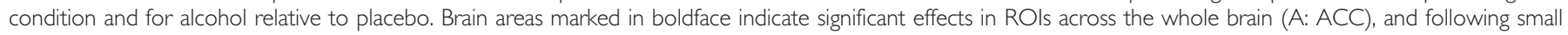
volume correction (SVC) (A: ACC; B: Putamen and Caudate). We report FWE-corrected and uncorrected $P$-values for the cluster and peak level, $t$-values, MNI

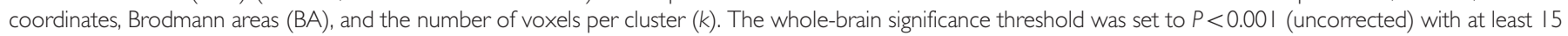
connected voxels.

${ }^{a} P=0.006$, FWE-corrected at peak level, following SVC across the ACC/medial PFC mask.

${ }^{\mathrm{b}} \mathrm{P}=0.004$, FWE-corrected at peak level, following SVC across the ventral striatum.

${ }^{c} P=0.033$, FWE-corrected at peak level, following SVC across the ventral striatum.

Neuropsychopharmacology 
Brain-behavior correlations in the amygdala differed only marginally significantly between provocation conditions at a corrected $\alpha$-level. Still, increased amygdala reactivity in participants exerting alcohol-induced reactive aggression, corresponds to its role in impulsive aggression (Raine et al, 1998; Meyer-Lindenberg et al, 2006; Coccaro et al, 2007). Our data do not support the assumption by Heinz et al (2011) that alcohol-induced changes of amygdala activation are mediated by impaired PFC functioning under alcohol as alcohol did not affect activation in the ACC/medial PFC. Probably alcohol does not affect evaluation-related medial PFC activity as behavioral provocation effects on aggression and RTs were present under alcohol as well as placebo. In contrast, alcohol decreased brain responses irrespective of provocation in inferior/middle frontal areas possibly reflecting impaired top-down control under alcohol (Heinz et al, 2011; Kareken et al, 2013; Gan et al, 2014).

The link between inter-individual levels of provocationinduced striatal and amygdala reactivity and the expression of aggressive behavior triggered by provocation further corroborates the role of the ventral striatum (Krämer et al, 2011; Beyer et al, 2014) and the amygdala in human reactive aggression (Coccaro et al, 2007). Thus inter-individual differences in provocation-induced ventral striatal and amygdala reactivity might be crucial for an individual disposition to express reactive aggression. It might further explain why group analyses revealed decreased instead of increased alcohol-related striatal activity, and neither main effects of alcohol or provocation in the amygdala. Moreover, neither provocation nor alcohol influenced neural circuits previously described in animal models of aggression, including the hypothalamus, periaqueductal gray, and ventral tegmental area (cf, Nelson and Trainor, 2007; Lin et al, 2011). Involvement of these areas in human alcoholinduced aggression remains to be elucidated.

Alcohol effects on BOLD responses reported in the present manuscript were not confounded by increased global perfusion under alcohol (Marxen et al, 2014), a possible interfering effect in pharmacological fMRI (Hoge et al, 1999), and are thus likely due to task-related changes of neural activity under alcohol and not to vascular changes. The absence of a gender effect on behavioral and neural alcohol effects of aggression further suggests that alcohol-induced aggression occurs in men as well as women (Dougherty et al, 1999; Giancola et al, 2009; Duke et al, 2011) but requires further investigation due to a small sample size.

For the first time, we show that neural and behavioral correlates of alcohol-induced aggression in humans vary as a function of provocation. Increased amygdala and ventral striatum reactivity under alcohol might represent a neural mechanism underlying an individual disposition for alcoholinduced reactive aggression typically elicited in stressful and threatening provoking situations (Heinz et al, 2011). In contrast, pronounced alcohol-induced proactive aggression might rely on the individual disposition to behave aggressively in a sober state but not on alcohol-induced changes of brain responses. Open questions emerge from the current research. First, the longitudinal D-LAYA study provides a unique opportunity to study whether neural and behavioral markers of a liability for alcohol-induced aggression are related to harmful alcohol use that may develop in some of our participants at ages 21-22 years (investigation under way). Future studies are needed to challenge the neural circuits of alcohol-induced aggression in clinical populations that are known to be associated with reactive aggression such as alcohol use disorders (Heinz et al, 2011), or intermittentexplosive disorder, and with proactive aggression such as anti-social personality disorder. In the long run, a better understanding of the neural mechanisms underlying alcoholinduced aggression in humans might contribute to the development of intervention methods (eg, anger management under alcohol) targeted at preventing 'brawls' in a bar and domestic violence.

\section{FUNDING AND DISCLOSURE}

The present work was supported by the National Institute on Alcohol Abuse and Alcoholism (NIAAA, Grant 1U01AA017900-01). Its contents are solely the responsibility of the authors and do not necessarily represent the official view of the NIAAA or NIH. This research was further supported by the Bundesministerium für Bildung und Forschung (BMBF Grant 01EV0711) and the Deutsche Forschungsgemeinschaft (DFG Grant: SM 80/7-1, ZI 1119/4-1, STE 1430/6-1, and SFB 940/1). GG was supported by the 'Gesellschaft von Freunden und Förderern der Technischen Universität Dresden e.V.' and the Graduate Academy of the TU Dresden during manuscript preparation. Over the past 3 years, USZ has received compensation from sächsische Landesärztekammer, Gewerkschaft Erziehung und Wissenschaft, Park-Krankenhaus Leipzig, ABW Wissenschaftsverlag, Servier, Janssen, and Lundbeck. The other authors declare no conflict of interest.

\section{ACKNOWLEDGMENTS}

We thank Professor Sean O'Connor for fruitful discussions during the setup of the study; Professor Dr Raffael Kalisch and Dr Andreas Marschner for technical advice of electronic stimulation during fMRI; Dr Mark J Jacob and Dr Amir H Javadi for help with constructing the ring electrode; the staff at the Neuroimaging Centre Dresden for managing MRI recordings, Eva Mennigen, and Maximilian Pilhatsch for medical supervision of experiments; Marie-Charlott Randhan, Daniel Schwarz, Zemfira Fechner, and Christine Markert for help during data acquisition and data quality control; Dr Kathrin U Müller and Dr Nelly Alia-Klein for fruitful discussions; and Dr. Nils B. Krömer for statistical advice. Parts of this work have been presented as posters at international conferences (Research Society on Alcoholism, 2013 and 2014; Society for Neuroscience, 2013; Society of Biological Psychiatry, 2014; Organization for Human Brain Mapping, 2014).

\section{REFERENCES}

Aharoni E, Vincent GM, Harenski CL, Calhoun VD, Sinnott-Armstrong W, Gazzaniga MS et al (2013). Neuroprediction of future rearrest. Proc Natl Acad Sci USA 110: 6223-6228.

Anderson BM, Stevens MC, Meda SA, Jordan K, Calhoun VD, Pearlson GD (2011). Functional imaging of cognitive control during acute alcohol intoxication. Alcohol Clin Exp Res 35: 156-165.

Bandura A (2001). Social cognitive theory: an agentic perspective. Annu Rev Psychol 52: 1-26. 
Beyer F, Munte TF, Kramer UM (2014). Increased neural reactivity to socio-emotional stimuli links social exclusion and aggression. Biol Psychol 96: 102-110.

Boileau I, Assaad JM, Pihl RO, Benkelfat C, Leyton M, Diksic M et al (2003). Alcohol promotes dopamine release in the human nucleus accumbens. Synapse 49: 226-231.

Bundeskriminalamt (2011). Polizeiliche Kriminalstatistik (PKS). Federal Ministry of the Interior (ed). IMK-Kurzbericht. www. bmi.bund.de.

Bureau of Justice Statistics (1998). Alcohol and Crime: An Analysis of National Data on the Prevalence of Alcohol Involvement in Crime. U.S. Department of Justice: Washington DC, USA.

Buss AH, Perry M (1992). The aggression questionnaire. J Pers Soc Psychol 63: 452-459.

Cherek DR, Steinberg JL, Manno BR (1985). Effects of alcohol on human aggressive-behavior. J Stud Alcohol 46: 321-328.

Coccaro EF, McCloskey MS, Fitzgerald DA, Phan KL (2007). Amygdala and orbitofrontal reactivity to social threat in individuals with impulsive aggression. Biol Psychiatry 62: 168-178.

de Wit H, Crean J, Richards JB (2000). Effects of d-amphetamine and ethanol on a measure of behavioral inhibition in humans. Behav Neurosci 114: 830-837.

Dichiara G, Imperato A (1986). Preferential stimulation of dopamine release in the nucleus-accumbens by opiates, alcohol, and barbiturates - studies with transcerebral dialysis in freely moving rats. Ann NY Acad Sci 473: 367-381.

Diedenhofen B. (2013). cocor: Comparing Correlations (Version 1.0-0). http://cran r-project org/web/packages/cocor/.

Dougherty DM, Bjork JM, Bennett RH, Moeller FG (1999). The effects of a cumulative alcohol dosing procedure on laboratory aggression in women and men. J Stud Alcohol 60: 322-329.

Duke AA, Giancola PR, Morris DH, Holt JCD, Gunn RL (2011). Alcohol dose and aggression: another reason why drinking more is a bad idea. J Stud Alcohol Drugs 72: 34-43.

Etkin A, Egner T, Kalisch R (2011). Emotional processing in anterior cingulate and medial prefrontal cortex. Trends Cogn Sci 15: 85-93.

Ferrari PF, van Erp AMM, Tornatzky W, Miczek KA (2003). Accumbal dopamine and serotonin in anticipation of the next aggressive episode in rats. Eur J Neurosci 17: 371-378.

Foran HM, O'Leary KD (2008). Alcohol and intimate partner violence: a meta-analytic review. Clin Psychol Rev 28: 1222-1234.

Gan G, Guevara A, Marxen M, Neumann M, Jünger E, Kobiella A et al (2014). Alcohol-induced impairment of inhibitory control is linked to attenuated brain responses in right frontotemporal cortex. Biol Psychiatry 76: 698-707.

Giancola PR, Godlaski AJ, Parrott DJ (2005). 'So I can't blame the booze?': dispositional aggressivity negates the moderating effects of expectancies on alcohol-related aggression. J Stud Alcohol 66: 815-824.

Giancola PR, Levinson CA, Corman MD, Godlaski AJ, Morris DH, Phillips JP et al (2009). Men and women, alcohol and aggression. Exp Clin Psychopharmacol 17: 154-164.

Giancola PR, Parrott DJ (2008). Further evidence for the validity of the Taylor Aggression Paradigm. Aggress Behav 34: 214-229.

Gilman JM, Ramchandani VA, Davis MB, Bjork JM, Hommer DW (2008). Why we like to drink: a functional magnetic resonance imaging study of the rewarding and anxiolytic effects of alcohol. J Neurosci 28: 4583-4591.

Gorka SM, Fitzgerald DA, King AC, Phan KL (2013). Alcohol attenuates amygdala-frontal connectivity during processing social signals in heavy social drinkers. A preliminary pharmacofMRI study. Psychopharmacology (Berl) 229: 141-154.

Heinz AJ, Beck A, Meyer-Lindenberg A, Sterzer P, Heinz A (2011). Cognitive and neurobiological mechanisms of alcohol-related aggression. Nat Rev Neurosci 12: 400-413.
Herzberg PY (2003). Faktorstruktur, gütekriterien und konstruktvalidität der deutschen übersetzung des aggressionsfragebogens von Buss und Perry. Z Differ Diagn Psychol 24: 311-323.

Hoge RD, Atkinson J, Gill B, Crelier GR, Marrett S, Pike GB (1999). Investigation of BOLD signal dependence on cerebral blood flow and oxygen consumption: the deoxyhemoglobin dilution model. Magn Reson Med 42: 849-863.

Kareken DA, Dzemidzic M, Wetherill L, Eiler W, Oberlin BG, Harezlak J et al (2013). Family history of alcoholism interacts with alcohol to affect brain regions involved in behavioral inhibition. Psychopharmacology (Berl) 228: 335-345.

Krämer UM, Jansma H, Tempelmann C, Munte TF (2007). Tit-for-tat: the neural basis of reactive aggression. Neuroimage 38: 203-211.

Krämer UM, Riba J, Richter S, Munte TF (2011). An fMRI study on the role of serotonin in reactive aggression. PLoS One 6: e27668.

Lieberman MD, Cunningham WA (2009). Type I and Type II error concerns in fMRI research: re-balancing the scale. Soc Cogn Affect Neurosci 4: 423-428.

Lin DY, Boyle MP, Dollar P, Lee H, Lein ES, Perona P et al (2011). Functional identification of an aggression locus in the mouse hypothalamus. Nature 470: 221.

Liu X, Hairston J, Schrier M, Fan J (2011). Common and distinct networks underlying reward valence and processing stages: a meta-analysis of functional neuroimaging studies. Neurosci Biobehav Rev 35: 1219-1236.

Maldjian JA, Laurienti PJ, Kraft RA, Burdette JH (2003). An automated method for neuroanatomic and cytoarchitectonic atlas-based interrogation of fMRI data sets. Neuroimage 19: $1233-1239$.

Marxen M, Gan G, Schwarz D, Mennigen E, Pilhatsch M, Zimmermann US et al (2014). Acute effects of alcohol on brain perfusion monitored with arterial spin labeling magnetic resonance imaging in young adults. J Cereb Blood Flow Metab 34: 472-479.

Meyer-Lindenberg A, Buckholtz JW, Kolachana B, Hariri AR, Pezawas L, Blasi G et al (2006). Neural mechanisms of genetic risk for impulsivity and violence in humans. Proc Natl Acad Sci USA 103: 6269-6274.

Miczek KA, Barros HM, Sakoda L, Weerts EM (1998). Alcohol and heightened aggression in individual mice. Alcohol Clin Exp Res 22: $1698-1705$.

Miczek KA, de Boer SF, Haller J (2013). Excessive aggression as model of violence: a critical evaluation of current preclinical methods. Psychopharmacology (Berl) 226: 445-458.

Miczek KA, Fish EW, de Bold JF, de Almeida RMM (2002). Social and neural determinants of aggressive behavior: pharmacotherapeutic targets at serotonin, dopamine and gamma-aminobutyric acid systems. Psychopharmacology (Berl) 163: 434-458.

Nelson RJ, Trainor BC (2007). Neural mechanisms of aggression. Nat Rev Neurosci 8: 536-546.

O'Connor S, Morzorati S, Christian J, Li TK (1998). Clamping breath alcohol concentration reduces experimental variance: application to the study of acute tolerance to alcohol and alcohol elimination rate. Alcohol Clin Exp Res 22: 202-210.

Parrott DJ, Zeichner A (2002). Effects of alcohol and trait anger on physical aggression in men. J Stud Alcohol 63: 196-204.

Raine A, Meloy JR, Bihrle S, Stoddard J, LaCasse L, Buchsbaum MS (1998). Reduced prefrontal and increased subcortical brain functioning assessed using positron emission tomography in predatory and affective murderers. Behav Sci Law 16: 319-332.

Seo D, Patrick CJ, Kennealy PJ (2008). Role of serotonin and dopamine system interactions in the neurobiology of impulsive aggression and its comorbidity with other clinical disorders. Aggress Violent Behav 13: 383-395.

Steiger JH (1980). Tests for comparing elements of a correlation matrix. Psychol Bull 87: 245-251. 
Sterzer P, Stadler C, Krebs A, Kleinschmidt A, Poustka F (2005). Abnormal neural responses to emotional visual stimuli in adolescents with conduct disorder. Biol Psychiatry 57: 7-15.

Taylor SP (1967). Aggressive behavior and physiological arousal as a function of provocation and the tendency to inhibit aggression. J Pers 35: 297-310.
Taylor SP, Gammon CB (1975). Effects of type and dose of alcohol on human physical aggression. J Pers Soc Psychol 32: 169-175.

Taylor SP, Gammon CB, Capasso DR (1976). Aggression as a function of interaction of alcohol and threat. J Pers Soc Psychol 34: 938-941.

Van Overwalle F (2009). Social cognition and the brain: a metaanalysis. Hum Brain Mapp 30: 829-858.

Supplementary Information accompanies the paper on the Neuropsychopharmacology website (http://www.nature.com/npp) 